\title{
EDS X-ray Characterization of S7 Tool Steel Laser Alloyed with Boron and Silicon
}

\author{
Justin D. Morrow $^{1 *}$, Stephen M. Seddio ${ }^{1}$ and Frank E. Pfefferkorn ${ }^{2}$ \\ 1. Thermo Fisher Scientific, Madison, WI USA. \\ 2. University of Wisconsin-Madison, Dept. of Mechanical Engineering, Madison, WI USA. \\ * Corresponding author: justin.morrow@thermofisher.com
}

Laser surface melting has been investigated as a final manufacturing step for smoothing [1], shaping [2], and chemically altering [3] the surface layer of metallic parts. Laser surface alloying is particularly interesting for the application of microscopy and microanalysis techniques, as it is critical to know how efficiently the alloying elements are melted into the surfaces (versus de-wetting or vaporizing). This study shows the challenges of such an analysis where the alloying elements to be added have a very different atomic mass compared to the base material and are deposited as thin films (up to 200-nmthick). The base material has several transition metals in the alloy with severely overlapping x-ray lines.

All characterization was done on a Zeiss LEO 1430 SEM, a windowless Thermo Scientific ${ }^{\text {TM }}$ UltraDry ${ }^{\mathrm{TM}}$ EDS detector $\left(30 \mathrm{~mm}^{2}\right.$ active area, $122.0 \mathrm{eV}$ resolution at Mn-K), and a Faraday cup for beam current measurement. Reference spectra were collected for B, C, Si, V, Cr, Mn, Fe, Mo from pure elemental standards blocks. The standards were cleaned prior to analysis by lightly polishing with $0.25 \mu \mathrm{m}$ diamond slurry and $\mathrm{MeOH}$ rinse, and then moved to the SEM for analysis. The detector position and sample working distance were held constant. Quantitative analyses were done at $15 \mathrm{kV}, 6400 \mathrm{~ns}$ shaping time, and $10 \%$ detector dead time with beam current measured prior to each EDS analysis.

A block of highly polished S7 steel ( $\mathrm{Ra} \sim 10 \mathrm{~nm}$ ) was sputter coated with overlapping layers of boron and silicon and laser melted, as shown in Fig 1(a) and (b). The film composition was validated with EDS at $3 \mathrm{kV}$ accelerating voltage and shown in Figure 1(c). The boron layer showed significant contribution from the steel substrate. This spectrum was well replicated by a simulation of a 40-nm-thick film of highly oxidized and lightly nitrided boron, done with DTSA-II software. Analysis of the S7 steel substrate (Fig. 1(c)) shows overlapping V, Cr, Fe, and Mn K- and L-lines, but standards-based quantitative analysis of this spectrum (Table 1) compares well to the expected values, indicating good peak deconvolution.

The main question, however, is whether the chemistry of the alloy was locally changed through thin film deposition and laser melting. Figure 2 shows the appearance of laser melted spots in the (a) Si-B-coatedS7, (b) B-coated-S7, (c) Si-coated-S7, and (d) uncoated-S7 steel regions along with overlay comparisons of the EDS spectra at the (e) boron and (f) silicon peak positions. A comparison of the quantified chemistry for the regions is given in Table 2. These results show that boron was negligible for all measurements, but the silicon content increased by a small but measurable amount from $0.45 \mathrm{wt} \%$ in the base material to $0.78 \mathrm{wt} \%$ in the Si-B-coated region and $1.47 \mathrm{wt} \%$ in the Si-coated region. This proves the fundamental feasibility of the laser alloying process, but also that it did not achieve the goal of creating a $\mathrm{Si}$ - and $\mathrm{B}$-rich surface layer. Interestingly, the quantitative analysis on the laser melted spots showed a decreased analytical total compared with analysis on the well-polished base material, potentially due to the micro-scale surface topography known to exist on the laser melted spots.

References: 
[1] Q Wang, JD Morrow, C Ma, N Duffie, FE Pfefferkorn, J. of Manuf. Proc. 20 (2015), p 340.

[2] FE Pfefferkorn and JD Morrow, CIRP Annals-Manuf. Tech. 66 (2017), p. 241.

[3] B Richter et al., J. of Manuf.Proc., 32 (2018), p. 230.

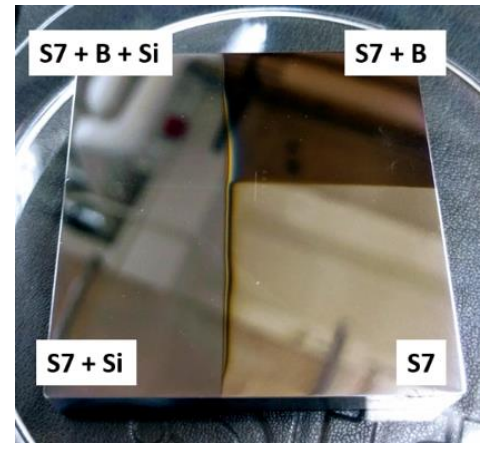

(a)

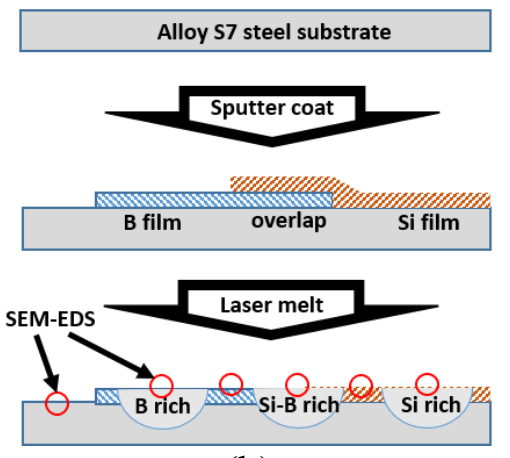

(b)

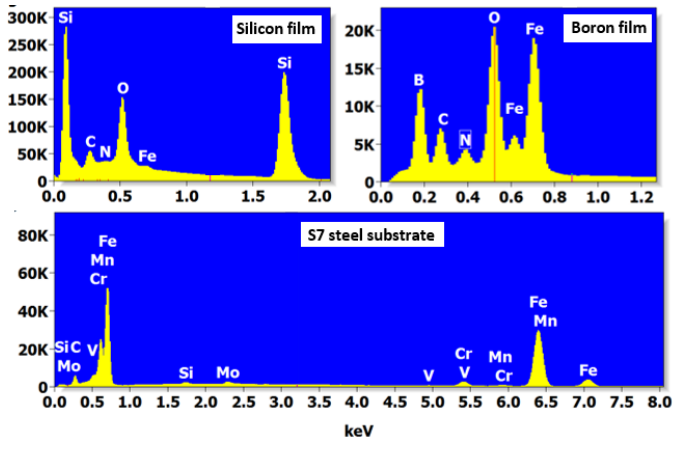

(c)

Figure 1. Orienting image (a) of coated steel block with (b) graphical processing description and (c) spectral analysis of coatings and base material. Sample aged 2 years in air and shows increased $\mathrm{O}$ and $\mathrm{N}$.

Table 1. Quantitative analysis of S7 substrate. ${ }^{\dagger}$ Carbon was set at fixed value due to contamination.

\begin{tabular}{|c|c|c|c|c|c|c|c|c|}
\hline & $\mathrm{C}$ & $\mathrm{Si}$ & $\mathrm{V}$ & $\mathrm{Cr}$ & $\mathrm{Mn}$ & $\mathrm{Fe}$ & Mo & Total \\
\hline Pathf & $0.5^{\dagger}$ & 0.67 & 0.26 & 3.71 & 0.78 & 995.64 & $\begin{array}{l}1.56 \\
\end{array}$ & 103.12 \\
\hline nictru (A S & $.45-0.55$ & $0.20-1.00$ & $0-0.35$ & $3.00-3.50$ & $0.20-0.90$ & Bal. & $1.30-1.80$ & 100 \\
\hline
\end{tabular}

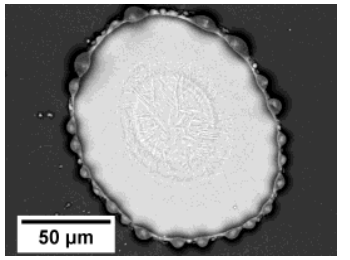

(a)

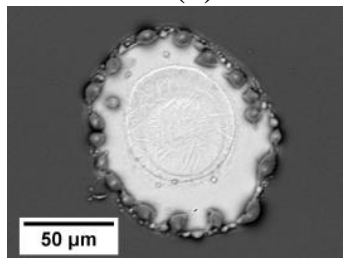

(c)

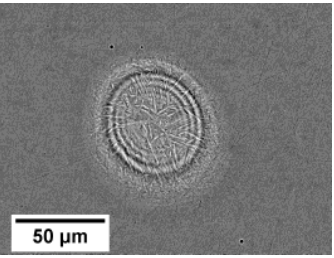

(b)

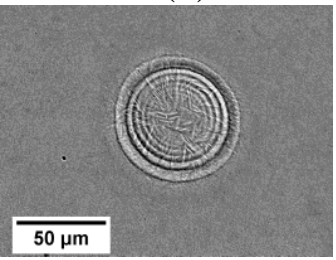

(d)

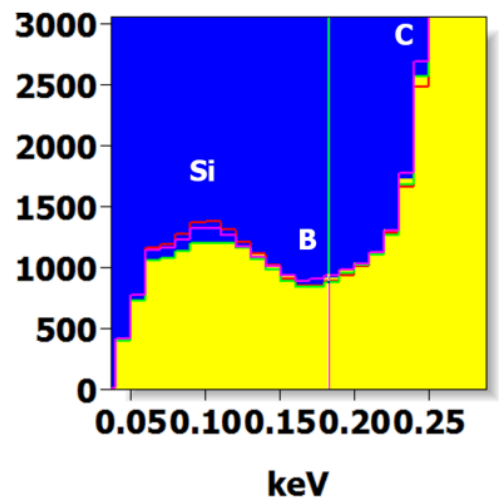

(e)

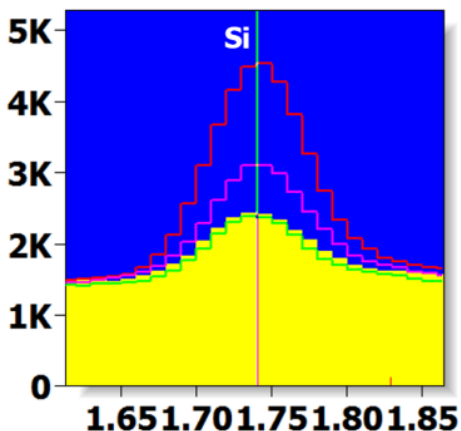

keV

(f)

Figure 2. SEM images of laser melted spots in the (a) Si-B-coated-S7, (b) B-coated-S7, (c) Si-coated-S7, and (d) uncoated-S7 steel regions with comparative overlays of the EDS spectra at the boron (e) and silicon (f) K-line peaks. Boron was negligible. Increased silicon is present in spectra from areas (a) and (c).

Table 2. Quantitative comparative analysis after laser melting. ${ }^{\dagger}$ Set at fixed value due to contamination.

\begin{tabular}{cccccccccc} 
Sample region & B & C & Si & V & Cr & Mn & Fe & Mo & Total \\
\hline \hline S7 (Fig2d) & 0.00 & $0.50^{\dagger}$ & 0.45 & 0.33 & 3.29 & 0.77 & 86.42 & 1.54 & $\mathbf{9 2 . 8 0}$ \\
S7 + B (Fig2b) & 0.00 & $0.50^{\dagger}$ & 0.45 & 0.22 & 3.15 & 0.70 & 86.01 & 1.33 & $\mathbf{9 1 . 8 6}$ \\
S7 + Si (Fig2c) & 0.00 & $0.50^{\dagger}$ & 1.47 & 0.30 & 3.25 & 0.66 & 88.32 & 1.41 & $\mathbf{9 5 . 4 1}$ \\
S7 + Si + B (Fig2a) & 0.00 & $0.50^{\dagger}$ & 0.78 & 0.24 & 3.34 & 0.39 & 87.86 & 1.68 & $\mathbf{9 4 . 2 9}$
\end{tabular}

